\title{
Diabetes mellitus, factor de riesgo de severidad de enfermedad isquémica crítica aterosclerótica y de viabilidad de miembros inferiores
}

\author{
Diabetes mellitus: a risk factor for severity of \\ critical atherosclerotic ischemic disease and for \\ lower limb viability
}

Octavio Martínez • Bogotá, Víctor Alfonso Castrillón • Pereira

\section{Resumen}

Objetivo: caracterizar la diabetes mellitus como factor de riesgo independiente para el grado de severidad de isquemia crítica de miembros inferiores y para la viabilidad de extremidades afectadas.

Contexto y tipo de estudio: censo de base hospitalaria sobre el registro de pacientes sometidos a revascularización o amputación primaria mayor de miembros inferiores por isquemia crítica aterosclerótica, a partir del primero de enero de 2005 hasta el 30 de junio de 2009. Las unidades de análisis fueron los registros clínicos de todos los pacientes intervenidos que cumplieran con los criterios de ingreso al estudio.

Material y métodos: descripciones univariada y bivariada dependiendo del tipo y distribución de las variables. Detección de variables confundidoras y con efectos de modificación de las medidas de asociación entre diabetes y severidad de isquemia crítica, y diabetes y tipo de intervención, mediante estratificación en el análisis.

Resultados: ajustada la relación para el efecto confundidor positivo de la hipertensión arterial, la posibilidad de diabetes mellitus en los pacientes Fontaine IV fue 8.23 veces la de los pacientes Fontaine III $\left(X^{2}{ }_{\text {MH 1gl }} 13.18\right.$; valor $\left.\mathrm{p}=0.0003\right)$. Controlado el efecto confundidor positivo de la insuficiencia renal crónica, la posibilidad de diabetes mellitus en los pacientes Fontaine IV fue 7.99 veces la de los pacientes Fontaine III $\left(X^{2}{ }_{\text {MH } 1 \mathrm{gl}} 12.00\right.$; valor $\left.\mathrm{p}=0.0005\right)$. Al controlar por la severidad de la isquemia crítica de miembros inferiores, los pacientes con diabetes mellitus conllevan una posibilidad de amputación primaria mayor de 6.32 en relación con los pacientes no diabéticos $\left(X_{\mathrm{MH} \text { 1gl }}^{2} 8.455\right.$; valor $\left.\mathrm{p}=0.0036\right)$.

Conclusión: la diabetes mellitus se señala como un factor de riesgo independiente para el desarrollo del estado más severo de isquemia crítica de miembros inferiores, Fontaine IV, tras ajustar para hipertensión arterial e insuficiencia renal crónica. Igualmente, la diabetes se constituyó en factor de riesgo independiente para la viabilidad de la extremidad con isquemia crítica (Acta Med Colomb 2010; 35: 40-47).

Palabras clave: diabetes mellitus, isquemia crítica miembros inferiores.

\footnotetext{
Abstract

Objective: to characterize diabetes mellitus as an independent risk factor for the severity of critical lower limb ischemia and for viability of affected limbs.

Context and type of study: Hospital based census on registers of all surgical patients for revascularization or major amputation by atherosclerotic critical ischemia or lower limbs from $1^{\text {st }}$ January, 2005 until $30^{\text {th }}$ June, 2009. Units of analysis were clinical records of patients who met the study's admission criteria.

Material and methods: univariate and bivariate descriptions depending on type and distribution of variables. Detection of confounders and modifiers of effects of the association between diabetes and severity of critical ischemia, and diabetes and intervention, by stratification in the analysis.
}

Dr. Octavio Martínez Betancur: Profesor Asociado, Facultad de Medicina, Universidad Nacional de Colombia, Bogotá; Dr. Víctor Alfonso Castrillón Muñoz: Cirujano General y Vascular, Sección de Cirugía Vascular y Angiología, Clínica Los Rosales. Pereira

Correspondencia. Dr. Octavio Martínez Betancur

E-mail: omartinezb@unal.edu.co Recibido: 05/V/10 Aceptado: 09/VI/10 
Results: adjusted for the positive confounding effect of hypertension, odds of diabetes mellitus in patients with ischemic disease Fontaine IV was 8.23 to patients with Fontaine III $\left(\mathrm{X}^{2}{ }_{\text {MH 1df }} 13.18 ; \mathrm{p}\right.$ value $=0.0003$ ). Controlled for the positive confounding effect of chronic renal failure, odds of diabetes mellitus in patients with Fontaine IV was 7.99 to patients with Fontaine III ( $\mathrm{X}^{2}{ }_{\text {MH ldf }} 12.00 ; \mathrm{p}$ value $=$ 0.0005). Controlled for the severity of critical lower limb ischemia, patients with diabetes mellitus had a possibility of primary major amputation 6.32 in relation to non-diabetic patients primary amputation $\left(\mathrm{X}_{\text {MH 1df }}^{2} 8.455 ; \mathrm{p}\right.$ value $\left.=0.0036\right)$.

Conclusion: Diabetes mellitus is an independent risk factor for the development of the most severe state of critical ischemia of the lower limbs, Fontaine IV, after adjusting for hypertension and chronic renal failure. Also, diabetes became an independent risk factor for the viability of the limb with critical ischemia. (Acta Med Colomb 2010; 35: 40-47)

Keywords. Diabetes mellitus, critical lower limb ischemia

\section{Introducción}

Independientemente del lecho vascular involucrado, la aterosclerosis es causada por un mismo proceso patológico. El proceso es diseminado y la enfermedad en un lecho vascular es una prueba sensible que marca la enfermedad en otro lecho vascular. Cuando el proceso aterotrombótico se manifiesta como enfermedad arterial obstructiva crónica (EAOC) de miembros inferiores (MMII), los factores de riesgo de los pacientes son similares a los encontrados en pacientes con aterosclerosis coronaria y cerebral (1-4).

La prevalencia de EAOC de MMII incrementa con la edad. El estudio de Framingham informa que las personas de 65 y más años, tienen un mayor riesgo de desarrollar EAOC de MMII. El informe NHANES (National Health and Nutrition Examination Survey, 1999 - 2000) (5) también anota una fuerte asociación entre la edad avanzada ( $\geq$ 70 años) y la prevalencia de EAOC de MMII: prevalencia de $4.3 \%$ en sujetos de 40 años y mayores, comparada con $14.5 \%$ en sujetos con 70 y más años.

La evidencia epidemiológica confirma mayor prevalencia de EAOC de MMII de causa aterosclerótica en pacientes diabéticos. El estudio de Framingham revela que $20 \%$ de los pacientes sintomáticos con EAOC de MMII tienen diabetes, y aclara que esta prevalencia puede estar subestimada porque muchas personas están asintomáticas; el verdadero estimativo de dicha prevalencia va de $30 \%$ a $40 \%(2,6)$. La duración y la severidad de la diabetes se correlacionan con la incidencia y la extensión de la EAOC de MMII, además de modificar la naturaleza de la EAOC. Los pacientes diabéticos tienen más comúnmente enfermedad arterial oclusiva infrapoplítea y calcificaciones vasculares que las cohortes no diabéticas, lo que limita la posibilidad de desarrollar circulación colateral y reduce las opciones de revascularización. Como consecuencia, es más probable que los pacientes diabéticos desarrollen formas más avanzadas de la enfermedad, convirtiéndose la diabetes en la causa número uno de amputaciones no traumáticas (7-10).

En pacientes con EAOC de causa aterosclerótica, las amputaciones no traumáticas de MMII son al menos 15 veces más prevalentes en pacientes diabéticos que en quienes cursan con cualquier otra condición médica concomitante
(11). El riesgo relativo para amputación en pacientes con diabetes es 12.7 (IC 95\%, 10.9 a 14.9) comparado con pacientes no diabéticos, y en pacientes diabéticos con edad entre 65 y 74 años, el riesgo relativo es tan alto como 23.5 (IC 95\%, 19.3 a 29.1) (7).

La diabetes no está sola como factor de riesgo en sus efectos sobre la vasculatura arterial periférica (6). Las personas con diabetes, en comparación con las no diabéticas, tienen mayor probabilidad de cursar con otros factores de riesgo adicionales para el desarrollo de EAOC, tales como tabaquismo, hipertensión, hiperlipidemia e insuficiencia renal crónica. También parece que tienen más inflamación vascular, disfunción de la célula endotelial y anormalidades de las células del músculo liso vascular, en comparación con los no diabéticos. Además, la diabetes se asocia con aumento de agregación plaquetaria y alteración de la función fibrinolítica (2).

La diabetes es un fuerte factor de riesgo para insuficiencia renal crónica (IRC) (11). La nefropatía diabética se desarrolla en $25 \%$ a $35 \%$ de pacientes con diabetes tipo 2, con la consecuente hipertensión que acarrea. Los pacientes con menores tasas de filtración glomerular tienen las mayores proporciones de diabetes mellitus e hipertensión (68\% a 79\%), con mayor frecuencia de isquemia crítica de MMII $(12,13)$.

Con respecto a IRC y amputación de MMII, aunque la mayoría de los estudios se enfoca en la enfermedad renal en estado terminal (prevalencia de EAOC entre $12 \%$ y $38 \%$ ) $(14,15)$, se aprecia una asociación con la EAOC aún en estados leves a moderados de insuficiencia renal $(11,14)$. El riesgo de amputación en pacientes diabéticos con IRC es al menos dos veces mayor que entre los que sólo tienen diabetes. La EAOC de MMII es el mecanismo que vincula la IRC con la amputación. El riesgo de amputación se asocia con la progresión de la IRC y es 10 veces el riesgo de pacientes sin enfermedad renal terminal, aun si se tiene en cuenta la diabetes $(11,16)$.

En pacientes con diabetes el riesgo de complicaciones vasculares se asocia fuertemente con hipertensión. La hipertensión se informa en $50 \%$ a $92 \%$ de los pacientes con EAOC de MMII. El estudio de Framingham demuestra un incremento entre $2.5 \mathrm{y} 4$ en el riesgo de desarrollar 
claudicación intermitente entre hombres y mujeres con hipertensión $(2,17)$.

El tabaquismo es el factor de riesgo modificable más importante para el desarrollo de EAOC de MMII y sus complicaciones: claudicación intermitente e isquemia crítica. La asociación entre tabaquismo y EAOC es casi dos veces tan fuerte como la encontrada entre tabaquismo y enfermedad coronaria (2).

La probabilidad de desarrollar EAOC aumenta en $10 \%$ por cada $10 \mathrm{mg} / \mathrm{dL}$ de aumento en el colesterol. Se acepta que el aumento del colesterol total, LDL y VLDL, y de los triglicéridos son todos factores de riesgo independientes para EAOC. La forma de dislipidemia que se diagnostica más frecuentemente en pacientes con EAOC es la combinación de un nivel reducido de HDL y un nivel elevado de triglicéridos, tal como se ve en pacientes con síndrome metabólico y diabetes (2).

La clasificación de Fontaine de los estados clínicos de la EAOC de MMII, establece: estado I, asintomático; estado II, claudicación; estado III, dolor en reposo durante mínimo dos semanas; estado IV, necrosis tisular (úlceras o gangrena). Poco se conoce acerca de la historia natural temprana de la EAOC, particularmente de la progresión de la enfermedad durante el periodo asintomático, y la transición hacia enfermedad sintomática temprana. A los estados III y IV de Fontaine se los denomina enfermedad con isquemia crítica de la extremidad, y se corresponden con estenosis únicas o múltiples de la luz de la arteria entre $50 \%$ y 99\%, u oclusión completa, con una incidencia poblacional estimada de 5.2 (IC $95 \% 4.3$ a 6.1) por 100 personas-año (18-21). La base para incluir los estados de Fontaine III y IV dentro de un mismo rubro denominado "isquemia crítica", es la presunción de una historia natural similar y de un pronóstico igualmente ominoso si dichas condiciones no son tratadas quirúrgicamente. Sin embargo, la "isquemia crítica" de miembros inferiores, no es una entidad nosológica homogénea ni es un proceso de enfermedad con varios grados de progresión, sino dos entidades clínicas distintivas con diferentes perfiles clínicos y diferente grado de severidad con perspectivas disímiles de viabilidad de la extremidad (22-27).

Este estudio parte de la hipótesis que la severidad y la viabilidad de extremidades inferiores (revascularización o amputación) con isquemia crítica de causa aterosclerótica, están determinados por la coexistencia o no de diabetes mellitus, y que tales efectos de isquemia tisular pueden estar mediados por otras condiciones clínicas crónicas coexistentes y relacionadas con la diabetes (tabaquismo, hipertensión arterial sistémica, hiperlipidemia, insuficiencia renal crónica), conocidas como factores de riesgo independientes para EAOC de MMII.

\section{Material y métodos}

Contexto y tipo de estudio

A partir de la base de datos clínica de la sección de Cirugía Vascular y Angiología de la Clínica Los Rosales de Pereira, base de datos computarizada donde se registran las historias clínicas de todos los pacientes intervenidos quirúrgicamente por patologías vasculares periféricas, arteriales y venosas, se realizó un censo de pacientes sometidos a revascularización o amputación primaria mayor de miembros inferiores por isquemia crítica aterosclerótica, a partir del primero de enero de 2005 hasta el 30 de junio de 2009. Las unidades de análisis fueron los registros clínicos de todos los pacientes intervenidos que cumplieran con los criterios de ingreso al estudio.

\section{Criterios de ingreso y de exclusión}

El criterio de ingreso al estudio fue un paciente intervenido quirúrgicamente para revascularización (angioplastia/ injerto venoso o protésico) o para amputación primaria mayor de MMII, por EAOC estados III y IV de Fontaine (isquemia crítica).

Como criterios de exclusión se consideraron: pacientes con amputación menor de MMII asociada o no con EAOC, pacientes con isquemia aguda de MMII y pacientes con procedimientos quirúrgicos relacionados con otras causas de úlceras crónicas y/o dolor en reposo de MMII (neuropatía sensorial no diabética, compresión radicular, enfermedad de Buerger, úlceras venosas, vasculitis, enfermedades del colágeno, úlceras asociadas con enfermedad hematológica, con trauma, infecciones, malignidad y gota). En los pacientes con revascularización o amputación del miembro inferior contralateral por isquemia crítica, y en los pacientes revascularizados con amputación subsecuente ipsilateral, sólo se consideró el primero de los procedimientos operatorios realizados (procedimiento quirúrgico incidente).

\section{Variables del estudio}

La edad de los pacientes fue consignada como años cumplidos al momento de la intervención quirúrgica. El sexo de los pacientes fue operacionalizado como variable dicotómica $(0=$ Mujer; $1=$ Hombre $)$.

El estado clínico de Fontaine, clasificatorio de la severidad de la enfermedad isquémica crítica de MMII, fue operacionalizado como variable dicotómica $(0=$ Fontaine III; 1 = Fontaine IV).

El tipo de intervención quirúrgica realizado, fue operacionalizado como variable dicotómica $(0=$ Revascularización; 1= Amputación).

Los diagnósticos de las siguientes condiciones crónicas fueron registrados a partir de lo consignado en las historias clínicas, y operacionalizados como variables dicotómicas $(0=\mathrm{No} ; 1=\mathrm{Si})$ :

- Diabetes mellitus (ICD 10 códigos E10, E11, E14, E10.2+);

- Insuficiencia renal crónica (ICD10 códigos N18.0, N18.9, N18.8);

- Hipertensión arterial sistémica, si se consignó como antecedente clínico en la historia, o si a la fecha de hospitalización se documentó tensión arterial igual o 
superior a 140/90 mmHg o si, aun con cifras tensionales normales, se encontraba consignada en la formulación médica terapia antihipertensiva;

- Hábito tabáquico, si se encontraba consignado el antecedente de fumador activo o pasado;

- Hiperlipidemia, si se documentaron niveles de colesterol total mayores a $200 \mathrm{mg} / \mathrm{dL}$, y/o colesterol LDL mayores a $100 \mathrm{mg} / \mathrm{dL}$ y/o se encontraba el antecedente de medicación hipolipemiante y/o se encontraba consignada en la formulación médica terapia hipolipemiante.

\section{Análisis estadístico}

La distribución de la edad se informa mediante valores mínimo, máximo y percentiles 25,50 y 75 . La edad fue categorizada con base en los cuartiles de su distribución para comparaciones con otras variables categóricas. Para la descripción de variables categóricas, se emplearon valores absolutos y relativos.

La prueba chi cuadrado $\left(X^{2}\right)$ de independencia de Pearson, se empleó para probar hipótesis de no asociación entre variables dicotómicas; en caso de que en una tabla de contingencia se superara $20 \%$ del total de frecuencias esperadas menores de cinco, se empleó la prueba exacta de Fisher para contrastar la hipótesis de independencia entre variables categóricas. La fuerza de la asociación entre variables dicotómicas, se evaluó mediante la oportunidad relativa (OR) y su intervalo de confianza al 95\% (IC 95\%).

El primer criterio para elegir entre las condiciones clínicas crónicas registradas, aquellas potenciales confundidoras de la medida del efecto causado por la diabetes mellitus sobre el estado clínico de isquemia crítica de MMII, fue su plausibilidad biológica, seguido del cumplimiento de distribución asimétrica entre los grupos de diabetes y no diabetes $\left(X^{2}\right.$ para diferencia de proporciones, valores $\left.\mathrm{p}<0.05\right)$. Seguidamente, se escogieron para análisis las variables que mostraran asociación positiva $(\mathrm{OR}>1)$ tanto con la diabetes como con el estado clínico de Fontaine (28-31).

Para determinar la homogeneidad de las ORs específicas de los estratos para cada una de las potenciales variables confundidoras, se empleó la prueba de Breslow-Day. En caso de determinarse homogeneidad de las ORs específicas de los estratos, se empleó la prueba de Cochran-Mantel-Haenszel para estimar la independencia condicional de la relación entre diabetes mellitus y la severidad de la enfermedad isquémica crítica de MMII. En caso de rechazarse la hipótesis nula de independencia condicional, y de considerarse homogeneidad de las ORs específicas de los estratos, se empleó la OR de Mantel-Haenszel para estimar la fuerza de la asociación entre diabetes mellitus y la severidad clínica de la isquemia crítica de MMII, ajustada para el efecto de cada una de las variables confundidoras del efecto. En caso de determinarse heterogeneidad de las ORs entre los estratos de potenciales variables confundidoras (modificación de la medida del efecto), solamente se informan los valores de las ORs crudas y específicas de los estratos (28-31).
Todas las pruebas estadísticas empleadas fueron de dos colas, con valor Alpha de 0.05 . Se emplearon los programas estadísticos SPSS versión 15 y Epidat versión 3.1.

\section{Resultados}

Del total de 136 historias clínicas de pacientes sometidos a intervenciones quirúrgicas en territorios vasculares arteriales, se censaron 72 pacientes que cumplieron con los criterios de inclusión. De las historias clínicas excluidas, 18 lo fueron por corresponder a procedimientos quirúrgicos sobre carótidas, 12 por ser procedimientos para corrección de trauma vascular, diez historias por tratarse de pacientes con aneurisma de aorta abdominal, diez por corresponder a intervenciones vasculares en pacientes con otros estados clínicos de Fontaine, seis historias por corresponder a eventos isquémicos agudos, cinco por ser intervenciones vasculares para inserción de catéteres y construcción de fístulas arteriovenosas, dos historias por tratarse de un paciente con vasculitis y otro con hipercoagulabilidad.

La edad mínima de los pacientes fue 40 años y la máxima 97 años; los percentil hombres, con una relación hombre: mujer de 1.12: 1 .

Los pacientes que cursaban con enfermedad isquémica crítica estado clínico Fontaine III fueron 27 (37.5\%) y con Fontaine IV, 45 (62.5\%) pacientes. Fueron 38 pacientes censados quienes tenían diabetes mellitus, y 34 pacientes sin diabetes. La asociación entre diabetes mellitus y severidad clínica de la enfermedad isquémica crítica de MMII fue estadísticamente significativa $\left(X^{2}{ }_{1 \mathrm{gl}}=20.344\right.$; valor $\mathrm{p}=$ 0.000 ) con una $\mathrm{OR}=12.10$ (IC 95\%, 3.74 a 39.16).

Todas las condiciones clínicas crónicas tenían plausibilidad biológica para ser estudiadas como potenciales confundidores del efecto de la diabetes mellitus sobre la severidad clínica de la isquemia crítica de MMII. Entre ellas, las que se distribuyeron asimétricamente en forma significativa entre los grupos de pacientes diabéticos y no diabéticos, fueron hipertensión arterial $\left(X^{2}{ }_{1 \mathrm{gl}}=20.33\right.$; valor $\mathrm{p}=0.000)$ e insuficiencia renal crónica $\left(X^{2}{ }_{1 \mathrm{gl}}=14.34\right.$; valor $\mathrm{p}=0.0001)$. La hipertensión arterial y la insuficiencia renal crónica mostraron asociación positiva $(\mathrm{OR}>1)$ de manera independiente, tanto con la diabetes mellitus como con el estado clínico de Fontaine.

La Tabla 1 muestra la segmentación de la relación entre diabetes mellitus y la severidad clínica de la isquemia crítica de MMII, con base en la variable hipertensión arterial, potencial confundidor de la relación. No se rechaza la hipótesis de homogeneidad de las ORs específicas de estratos, y se rechaza la hipótesis de independencia condicional. Implica que la hipertensión arterial no modifica la fuerza de la medida del efecto de la diabetes mellitus sobre la severidad de la isquemia crítica, pero sí ejerce un efecto confundidor positivo sobre la relación, efecto para controlar. Una vez ajustada la relación para el efecto confundidor de la hipertensión arterial, la posibilidad de diabetes mellitus en los pacientes Fontaine IV fue 8.23 
Tabla 1. Segmentación de la relación entre diabetes mellitus y severidad de la isquemia crítica de MMII, con base en la hipertensión arterial.

\begin{tabular}{|c|c|c|c|c|}
\hline \multirow{2}{*}{$\begin{array}{l}\text { Hipertensión } \\
\text { arterial }\end{array}$} & \multirow{2}{*}{$\begin{array}{l}\text { Diabetes } \\
\text { mellitus }\end{array}$} & \multicolumn{2}{|c|}{ Isquemia crítica } & \multirow[t]{2}{*}{ Tota } \\
\hline & & Fontaine IV & Fontaine III & \\
\hline No & $\begin{array}{c}\text { Si } \\
\text { No } \\
\text { Total }\end{array}$ & $\begin{array}{l}2 \\
6 \\
8\end{array}$ & $\begin{array}{c}1 \\
12 \\
13\end{array}$ & $\begin{array}{c}3 \\
18 \\
\mathbf{2 1}\end{array}$ \\
\hline \multicolumn{5}{|l|}{$\mathrm{OR}=4.00$} \\
\hline $\mathrm{Si}$ & $\begin{array}{c}\mathrm{Si} \\
\text { No } \\
\text { Total }\end{array}$ & $\begin{array}{c}31 \\
6 \\
37\end{array}$ & $\begin{array}{c}5 \\
10 \\
15\end{array}$ & $\begin{array}{l}36 \\
16 \\
\mathbf{5 2}\end{array}$ \\
\hline \multicolumn{5}{|c|}{$\begin{array}{l}\text { OR }=10.33 \\
\text { OR cruda }=10.08 \\
\text { Prueba de Breslow-Day: } \mathrm{X}^{2}{ }_{\text {Igl }}=0.411 ; \mathrm{p}=0.522 \\
\text { Prueba de Cochran-Mantel-Haenszel: } \mathrm{X}^{2}{ }_{\mathrm{glg}}=13.53 ; \mathrm{p}=0.000 \\
\text { OR de Mantel-Haenszel }=8.23(\mathrm{IC} 95 \%, 2.43 \text { a } 27.86)\end{array}$} \\
\hline
\end{tabular}

veces la de los pacientes Fontaine III $\left(X^{2}{ }_{\mathrm{MH} 1 \mathrm{gl}} 13.18\right.$; valor $\mathrm{p}=0.0003)$.

La Tabla 2 muestra la segmentación de la relación entre diabetes mellitus y la severidad clínica de la isquemia crítica de MMII, con base en la variable insuficiencia renal crónica, potencial confundidor de la relación. Se concluye de los estimativos de la Tabla 2, que la insuficiencia renal crónica ejerce un efecto confundidor positivo sobre la relación entre diabetes mellitus y severidad de la isquemia crítica de MMII, y que una vez controlado dicho efecto confundidor, la posibilidad de diabetes mellitus en los pacientes Fontaine IV fue 7.99 veces la de los pacientes Fontaine III $\left(X^{2}{ }_{\mathrm{MH} \mathrm{lgl}}\right.$ 12.00; valor $\mathrm{p}=0.0005$ ).

De las 44 amputaciones primarias mayores de MMII, sólo una fue realizada por debajo de la rodilla; las demás fueron amputaciones supracondíleas. La Tabla 3 comparó el tipo de intervención quirúrgica realizada en función del estado clínico de Fontaine, y muestra que la mayor frecuencia de amputaciones se realizó en los pacientes con enfermedad más severa Fontaine IV, mientras que las revascularizaciones de salvamento de la extremidad se ofrecieron más frecuentemente a pacientes sin pérdida tisular, con diferencia estadísticamente significativa $\left(X^{2}{ }_{1 \mathrm{gl}}\right.$ Pearson $=27.49$; valor $\mathrm{p}=0.000)$. Se concluye de la Tabla 3 que la posibilidad de amputación para los pacientes Fontaine IV es 19 veces la de los pacientes Fontaine III. Por su parte, la posibilidad de amputación primaria mayor para pacientes diabéticos es 13.8 veces la de los pacientes no diabéticos $\left(X^{2}{ }_{191}\right.$ Pearson $=22.42$; valor $\mathrm{p}=0.000$ ) Tabla 4 .

La Tabla 5 muestra la segmentación de la relación entre diabetes mellitus y el tipo de intervención quirúrgica realizada, controlado por el estado clínico de Fontaine. El estado clínico de Fontaine es un confundidor positivo del efecto de la diabetes mellitus sobre el tipo de intervención quirúrgica. $\mathrm{Al}$ controlar por la severidad de la isquemia crítica de MMII, los pacientes con diabetes mellitus conllevan una posibilidad de amputación primaria mayor de 6.32 en relación con los pacientes no diabéticos $\left(X^{2}{ }_{\text {MH } 1 \mathrm{gl}} 8.455\right.$; valor $\left.\mathrm{p}=0.0036\right)$. Por su parte, cuando se controla la relación entre diabetes
Tabla 2. Segmentación de la relación entre diabetes mellitus y severidad de la isquemia crítica de MMII, con base en insuficiencia renal crónica.

\begin{tabular}{|c|c|c|c|c|}
\hline \multirow{2}{*}{$\begin{array}{l}\text { Insuficiencia } \\
\text { renal crónica }\end{array}$} & \multirow{2}{*}{$\begin{array}{l}\text { Diabetes } \\
\text { mellitus }\end{array}$} & \multicolumn{2}{|c|}{ Isquemia crítica } & \multirow[t]{2}{*}{ Total } \\
\hline & & Fontaine IV & Fontaine III & \\
\hline No & $\begin{array}{c}\mathrm{Si} \\
\text { No } \\
\text { Total }\end{array}$ & $\begin{array}{l}15 \\
10 \\
25\end{array}$ & $\begin{array}{c}4 \\
21 \\
25\end{array}$ & $\begin{array}{l}19 \\
31 \\
\mathbf{5 0}\end{array}$ \\
\hline \multicolumn{5}{|l|}{$\mathrm{OR}=7.88$} \\
\hline $\mathrm{Si}$ & $\begin{array}{c}\text { Si } \\
\text { No } \\
\text { Total }\end{array}$ & $\begin{array}{c}18 \\
2 \\
20\end{array}$ & $\begin{array}{l}1 \\
1 \\
2\end{array}$ & $\begin{array}{c}19 \\
3 \\
22\end{array}$ \\
\hline \multicolumn{5}{|c|}{$\begin{array}{l}\text { OR }=9.00 \\
\text { OR cruda }=12.10 \\
\text { Prueba de Breslow-Day: } X^{2}{ }_{1 \mathrm{gl}}=0.006 ; \mathrm{p}=0.938 \\
\text { Prueba de Cochran-Mantel-Haenszel: } X^{2}{ }_{\text {lel }}=12.27 ; \mathrm{p}=0.000 \\
\text { OR de Mantel-Haenszel }=7.99(\mathrm{IC} 95 \%, 2.31 \text { a } 27.60)\end{array}$} \\
\hline
\end{tabular}

Tabla 3. Relación del tipo de intervención quirúrgica en función del estado clínico de Fontaine.

\begin{tabular}{|c|c|c|}
\hline \multirow[t]{2}{*}{ Estado clínico } & \multicolumn{2}{|c|}{ Tipo de intervención } \\
\hline & $\begin{array}{r}\text { Amputación } \\
\mathbf{N}(\%)\end{array}$ & $\begin{array}{r}\text { Revascularización } \\
\mathbf{N}(\%)\end{array}$ \\
\hline $\begin{array}{r}\text { Fontaine IV } \\
\text { Fontaine III } \\
\text { Total }\end{array}$ & $\begin{array}{r}38(86.40) \\
6(13.60) \\
44 \quad(100)\end{array}$ & $\begin{array}{r}7(25.00) \\
21(75.00) \\
28 \quad(100)\end{array}$ \\
\hline
\end{tabular}

Tabla 4. Relación del tipo de intervención quirúrgica en función de la condición clínica diabetes mellitus.

\begin{tabular}{|c|c|c|}
\hline \multirow[t]{2}{*}{ Diabetes mellitus } & \multicolumn{2}{|c|}{ Tipo de intervención } \\
\hline & $\begin{array}{c}\text { Amputación } \\
\text { N (\%) }\end{array}$ & $\begin{array}{c}\text { Revascularización } \\
\text { N }(\%)\end{array}$ \\
\hline $\begin{array}{r}\text { Si } \\
\text { No } \\
\text { Total }\end{array}$ & $\begin{array}{c}33(75.00) \\
11(25.00) \\
\mathbf{4 4 ( 1 0 0 )}\end{array}$ & $\begin{array}{l}5(17.85) \\
23(82.15) \\
\mathbf{2 8} \quad(\mathbf{1 0 0})\end{array}$ \\
\hline $\mathrm{OR}=13.80(\mathrm{IC} 95 \%, 4.22$ & & \\
\hline
\end{tabular}

Tabla 5. Segmentación de la relación entre la condición clínica diabetes mellitus y el tipo de intervención quirúrgica, con base en el estado clínico de Fontaine.

\begin{tabular}{|c|c|c|c|c|}
\hline \multirow{2}{*}{$\begin{array}{l}\text { Estado } \\
\text { clínico }\end{array}$} & \multirow{2}{*}{$\begin{array}{l}\text { Diabetes } \\
\text { mellitus }\end{array}$} & \multicolumn{2}{|c|}{ Intervención quirúrgica } & \multirow[t]{2}{*}{ Total } \\
\hline & & Amputación & Revascularización & \\
\hline Fontaine III & $\begin{array}{c}\mathrm{Si} \\
\text { No } \\
\text { Total }\end{array}$ & $\begin{array}{l}2 \\
4 \\
6\end{array}$ & $\begin{array}{c}3 \\
18 \\
\mathbf{2 1}\end{array}$ & $\begin{array}{c}5 \\
22 \\
\mathbf{2 7}\end{array}$ \\
\hline \multicolumn{5}{|l|}{$\mathrm{OR}=3.00$} \\
\hline Fontaine IV & $\begin{array}{c}\mathrm{Si} \\
\text { No } \\
\text { Total }\end{array}$ & $\begin{array}{c}31 \\
7 \\
38\end{array}$ & $\begin{array}{l}2 \\
5 \\
7\end{array}$ & $\begin{array}{l}33 \\
12 \\
45\end{array}$ \\
\hline \multicolumn{5}{|c|}{$\begin{array}{l}\text { OR }=11.10 \\
\text { OR cruda }=13.80 \\
\text { Prueba de Breslow-Day: } X^{2}{ }_{1 \mathrm{gl}}=0.861 ; \mathrm{p}=0.353 \\
\text { Prueba de Cochran-Mantel-Haenszel: } X^{2}{ }^{1 \mathrm{gl}}=8.697 ; \mathrm{p}=0.003 \\
\text { OR de Mantel-Haenszel }=6.32(\text { IC } 95 \%, 1.67 \mathrm{a} 23.92)\end{array}$} \\
\hline
\end{tabular}


mellitus y el tipo de intervención quirúrgica realizada por la condición clínica de insuficiencia renal crónica, otro factor pronóstico de amputación en isquemia crítica de MMII, los pacientes diabéticos tienen una posibilidad de ser amputados de 7.80 (IC 95\%, 2.25 a 27.05) en relación con los no diabéticos (datos no mostrados).

\section{Discusión}

Puesto que la enfermedad arterial periférica no clasifica entre las primeras causas de mortalidad, los estudios epidemiológicos que la abordan se han dedicado a investigar factores de riesgo cardiovascular implicados en la etiología de la enfermedad cardiaca coronaria, con orfandad de evidencia clínica para caracterizar la enfermedad oclusiva crónica periférica de causa aterosclerótica. La evidencia producida indica que los mismos factores de riesgo para enfermedad coronaria también son importantes cuando la enfermedad aterosclerótica se presenta primariamente en las arterias periféricas. Existe, sin embargo, disputa entre datos epidemiológicos sobre algunas diferencias en el perfil de los factores de riesgo, en relación con los diferentes grados de severidad del compromiso aterosclerótico en los diferentes territorios vasculares (32-40).

La diabetes mellitus se percibe como un factor de riesgo importante para enfermedad vascular periférica, un tanto más que para enfermedad cardiaca coronaria. Este tanto más de riesgo hace referencia a la enfermedad isquémica crítica de MMII en la cual, además de la aterosclerosis, otros factores tienen efectos. La diabetes también causa microangiopatía, neuropatía con la pérdida de sensibilidad y el daño tisular traumático, y un aumento de riesgo para la infección. Todos estos factores aumentan el riesgo de gangrena y ulceración, y la necesidad de amputación (41-43).

En el presente estudio, la diabetes mellitus se señala como un factor de riesgo independiente para el desarrollo del estado más severo de isquemia crítica de MMII, Fontaine IV, tras ajustar para hipertensión arterial e insuficiencia renal crónica, dos factores de riesgo conocidos, además de la diabetes, para el desarrollo de aterosclerosis sistémica. Igualmente, la diabetes se constituyó en factor pronóstico independiente para la viabilidad de la extremidad con isquemia crítica, aparte de otros dos factores que determinan también la posibilidad de amputación, el estado clínico de severidad de Fontaine y la insuficiencia renal crónica.

El estudio de Jude y col. (44), diseñado para cuantificar la distribución de enfermedad arterial periférica en pacientes diabéticos y no diabéticos, además de la severidad de la enfermedad vascular y el desenlace entre los dos grupos de pacientes, informa que aunque los pacientes diabéticos tuvieron mayor frecuencia de úlceras y gangrena de la extremidad que los no diabéticos, el dolor en reposo fue menos común en pacientes diabéticos; los pacientes diabéticos, no obstante, cursaron con mayor proporción de hipertensión. Mediante angiografías y con base en el sistema de puntuación de Bollinger, el mismo estudio establece que los pacientes diabéticos tuvieron enfermedad arterial más severa por debajo de la rodilla que los no diabéticos. Por otra parte, los pacientes diabéticos tuvieron cinco veces más posibilidad que los pacientes no diabéticos de ser amputados. Enfatizar que el diseño del estudio de Jude y col. fue transversal, de base hospitalaria y sin ajuste para posibles confundidores de efecto.

El estudio de Rotherdam (40) un estudio prospectivo de cohorte de base poblacional, orientado a investigar la incidencia y determinantes de enfermedades crónicas discapacitantes, encuentra la diabetes mellitus como un predictor fuerte sólo de progresión severa de aterosclerosis (ni progresión leve ni moderada), específicamente aórtica, sin interacción estadísticamente significativa con otros factores de riesgo.

Abou-Zamzam y col. (45), realizan un estudio prospectivo de seguimiento durante cuatro años de pacientes con isquemia crítica de MMII, y examina los factores que conducen a amputación primaria versus revascularización; mediante análisis multivariable establecen como predictores independientes de amputación primaria mayor, la pérdida de tejido (Fontaine IV), la enfermedad renal terminal, la diabetes mellitus y el estado no ambulatorio del paciente. Cincuenta y cuatro por ciento de todas las amputaciones primarias mayores se realizaron debido a gangrena extensa o infección presentes al momento de la evaluación vascular general, lo que limitó la recuperación de la extremidad.

En el estudio de Virkkunnen y col. (46), estudio fundamentado en el registro nacional finlandés con datos de nueve años y realizado para analizar la significación de la diabetes como factor de riesgo para mortalidad y morbilidad posoperatoria temprana en reconstrucciones vasculares, la manifestación más común de isquemia crítica de MMII fue la ulceración (51.6\%), seguida de dolor en reposo (37.9\%) y gangrena (10.5\%), en un total de 5709 intervenciones quirúrgicas por isquemia crítica. Como indicación quirúrgica, la ulceración y la gangrena fueron más frecuentes en los pacientes diabéticos en comparación con los no diabéticos. Por otra parte, la tasa de revascularización para pacientes diabéticos con isquemia crítica fue más baja que para pacientes no diabéticos, y la diabetes se constituyó en un factor de riesgo independiente para amputación y complicaciones en isquemia crítica de la extremidad.

Aún no acaba la discusión académica sobre los objetivos a lograr tras la decisión quirúrgica a seguir en un paciente con enfermedad isquémica crítica de MMII. Los objetivos instituidos por consensos de grupos de estudio sobre el tema, son puestos cada vez más en entredicho $(22,47-49)$. Se tiene claro que las decisiones se ven influenciadas por prácticas locales y actitudes clínicas parcialmente subjetivas, de tal suerte que la amputación puede verse como parte de la aproximación al manejo paliativo del dolor y la infección. Aunque es difícil establecer si la práctica de amputaciones en el presente estudio se ajusta a decisiones clínicas consecuentes con el estado de la enfermedad, es muy diciente 
que así sea, puesto que del total de pacientes diabéticos (38/72), fueron amputados 33 pacientes, y de estos últimos, 31 cursaban con enfermedad Fontaine IV.

Tampoco existe acuerdo sobre la definición de una extremidad isquémica no rescatable, pero se identifican indicaciones para pacientes con isquemia crítica de MMII que se beneficiarían de amputación primaria mayor, a menudo con regreso a condiciones de vida satisfactorias y útiles: enfermedad oclusiva arterial que no permita reconstrucción, necrosis de áreas significativas de las porciones de apoyo del pie, contractura fija en flexión de la pierna, enfermedad terminal, o una muy limitada expectativa de vida debido a condiciones comórbidas (26).

La evidencia epidemiológica recabada con diferentes diseños de estudio, en diferentes contextos, con diferentes medidas de desenlace, con ajustes o no para confundidores, es consistente con los hallazgos aquí informados, lo que fortalece la inferencia causal de la diabetes mellitus sobre la severidad de la enfermedad isquémica crítica de MMII y sobre la viabilidad de la extremidad afectada (50).

\section{Referencias}

1. Leng GC, Lee AJ, Fowkes FG, Whiteman M, Dunbar J, Housley E, et al. Incidence, natural history and cardiovascular events in symptomatic and asymptomatic peripheral arterial disease in the general population. Int J Epidemiol 1996; 25: $1172-81$

2. Duvall WL, Vorchheimer DA. Multi-bed vascular disease and atherothrombosis: scope of the problem. J Thromb Thrombolysis 2004; 17: 51-61.

3. Vidakovic R, Schouten O, Kuiper R, Hoeks SE, Flu WJ, van Kuijk JP, et al. The prevalence of polyvascular disease in patients referred for peripheral arterial disease. Eur J Vasc Endovasc Surg 2009; 38: 435-40.

4. Bartholomew JR, Olin JW. Pathophysiology of peripheral arterial disease and risk factors for its development. Cleve Clin J Med 2006; 73: S8-S14.

5. Selvin E, Erlinger TP. Prevalence of and risk factors for peripheral arterial disease in the United States: results from the National Health and Nutrition Examination Survey, 1999-2000. Circulation 2004; 110: 738-43.

6. Sheehan P. Peripheral arterial disease in patients with diabetes. En: Abela GS. Peripheral vascular disease: basic diagnostic and therapeutic approaches. Philadelphia: Lippincot Williams \& Wilkins; 2004. Electronic edition.

7. Mowla A, Bahrami A, Dastgheib SA. Peripheral arterial disease in patients with diabetes mellitus. South Med J 2006; 99: 707-8.

8. Bembi V, Singh S, Singh P, Aneja GK, Arya TV, Arora R. Prevalence of peripheral arterial disease in a cohort of diabetic patients. South Med J 2006; 99: 564-9.

9. Leibson CL, Ransom JE, Olson W, Zimmerman BR, O'fallon WM, Palumbo PJ. Peripheral arterial disease, diabetes, and mortality. Diabetes Care 2004; 27: 2843-9.

10. Lüscher TF, Creager MA, Beckman JA, Cosentino F. Diabetes and vascular disease: pathophysiology, clinical consequences, and medical therapy: part II. Circulation 2003; 108: 1655-61.

11. Margolis DJ, Hofstad O, Feldman HI. Association between renal failure and foot ulcer or lower-extremity amputation in patients with diabetes. Diabetes Care 2008; 31: 1331-6.

12. Owens CD, Ho KJ, Kim S, Schanzer A, Lin J, Matros E, et al. Refinement of survival prediction in patients undergoing lower extremity bypass surgery: stratification by chronic kidney disease classification. J Vasc Surg 2007; 45: 944-52.

13. Joss N, Paterson KR, Deighan CJ, Simpson K, Boulton-Jones JM. Vascular disease and survival in patients with type 2 diabetes and nephropathy. $\mathrm{Br} J$ Diabetes \& Vasc Dis 2002; 2: 137-42.

14. O’Hare AM, Glidden DV, Fox CS, Hsu CY. High prevalence of peripheral arterial disease in persons with renal insufficiency. Results from the National Health and Nutrition Examination Survey 1999 - 2000. Circulation 2004; 109: 320-3.

15. Rajagopalan S, Dellegrottaglie S, Furniss AL, Gillespie BW, Satayathum $\mathbf{S}$, Lameire N, et al. Peripheral arterial disease in patients with end-stage renal disease: observations from the Dyalisis Outcomes and Practice Patterns Study (DOPPS). Circulation 2006; 114: 1914-22.
16. Boufi M, Ghaffari P, Allaire E, Fessi H, Ronco P, Vayssairat M. Foot gangrene in patients with end-stage renal disease: a case control study. Angiology 2006; 57: $355-61$

17. Adler AI, Stratton IM, Neil HA, Yudkin JS, Matthews DR, Cull CA, et al. Association of systolic blood pressure with macrovascular and microvascular complications of type 2 diabetes (UKPDS 36): prospective observational study. BMJ 2000; 321: 412-9.

18. Hankey GJ, Norman PE, Eikelboom JW. Medical treatment of peripheral arterial disease. JAMA 2006; 295: 547-53.

19. Nehler MR, Hiatt WR, Taylor LM Jr. Is revascularization and limb salvage always the best treatment for critical limb ischemia? J Vasc Surg 2003; 37: 704-8.

20. Sottiurai V, White JV. Extensive revascularization or primary amputation: which patients with critical limb ischemia should not be revascularized? Semin Vasc Surg 2007; 20: 68-72.

21.Pedrini L. Critical ischaemia of the lower limbs: diagnostic and therapeutic strategies. Foot Ankle Surg 2003; 9: 87-94.

22. Taylor SM, Cull DL, Kalbaugh CA, Senter HF, Langan EM 3rd, Carsten CG 3rd, et al. Comparison of interventional outcomes according to preoperative indication: a single center analysis on 2,240 limb revascularizations. J Am Coll Surg 2009; 208: 770-80.

23. Hirsch AT, Haskal ZJ, Hertzer NR, Bakal CW, Creager MA, Halperin JL, et al. ACC/AHA 2005 Guidelines for the management of patients with peripheral arterial disease (lower extremity, renal, mesenteric, an abdominal aortic): executive summary a collaborative report from the American Association for Vascular Surgery/Society for Vascular Surgery, Society for Cardiovascular Angiography and Interventions, Society for Vascular Medicine and Biology, Society of Interventional Radiology, and the ACC/AHA Task Force on Practice Guidelines (Writing Committee to Develop Guidelines for the Management of Patients With Peripheral Arterial Disease) endorsed by the American Association of Cardiovascular and Pulmonary Rehabilitation; National Heart, Lung, and Blood Institute; Society for Vascular Nursing; TransAtlantic Inter-Society Consensus; and Vascular Disease Foundation. J Am Coll Cardiol 2006; 47: 1239-312.

24. Norgren L, Hiatt WR, Dormandy JA, Nehler MR, Harris KA, Fowkes FG; TASC II Working Group. Inter-Society Consensus for the Management of Peripheral Arterial Disease (TASC II). J Vasc Surg 2007; 45: S5-S67.

25. Lepäntalo M, Mätzke S. Outcome of unreconstructed chronic critical leg ischaemia. Eur J Vasc Endovasc Surg 1996; 11: 153-7.

26. Varu VN, Hogg ME, Kibbe MR. Critical limb ischemia. J Vasc Surg 2010; 51: 230-41.

27. American Diabetes Association. Peripheral arterial disease in people with diabetes. Diabetes Care 2003; 26: 3333-41.

28. Londoño JL. Metodología de la investigación epidemiológica. 3a. ed. Bogotá Editorial Manual Moderno; 2004

29. Szklo M, Nieto J. Epidemiología intermedia: conceptos y aplicaciones. 1a. ed. Madrid: Editorial Díaz de Santos; 2003.

30. Oleckno WA. Epidemiology. Concepts and methods. 1a. ed. Illinois: Editorial Waveland Pr Inc; 2008

31. Jewell NP. Statistics for epidemiology. 1a. ed. Boca Raton: Editorial Chapman and Hall/CRC; 2003.

32. Nair DG, Samson R. Which diabetics are at risk for lower-extremity problems and what preventive measures can be taken?. Sem Vasc Surg 2008; 21: 154-9.

33. Marso SP, Hiatt WR. Peripheral arterial disease in patients with diabetes. $J$ Am Coll Cardiol 2006; 47: 921-9.

34. Wattanakit K, Folsom AR, Selvin E, Weatherley BD, Pankow JS, Brancat FL, et al. Risk factors for peripheral arterial disease incidence in persons with diabetes: the Atherosclerosis Risk in Communities (ARIC) Study. Atherosclerosis 2005; 180: 389-97.

35. Mackaay AJ, Beks PJ, Dur AH, Bischoff M, Scholma J, Heine RJ, et al. The distribution of peripheral vascular disease in a Dutch Caucasian population: comparison of type II diabetic and non-diabetic subjects. Eur J Vasc Endovasc Surg 1995; 9: 170-5.

36. Golomb BA, Dang TT, Criqui MH. Peripheral arterial disease: morbidity and mortality implications. Circulation 2006; 114: 688-99.

37. Monreal M, Alvarez L, Vilaseca B, Coll R, Suárez C, Toril J, et al. Clinical outcome in patients with peripheral artery disease. Results from a prospective registry (FRENA). Eur J Intern Med 2008; 19: 192-7.

38. Wassink AM, van der Graaf Y, Olijhhoek JK, Visserin FL; SMART Study Group. Metabolic syndrome and the risk of new vascular events and all-cause mortality in patients with coronary artery disease, cerebrovascular disease, peripheral arterial disease or abdominal aortic aneurysm. Eur Heart J 2008; 29: 213-23.

39. Qadan LR, Ahmed AA, Safar HA, Al-Bader MA, Ali AA. Prevalence of metabolic syndrome in patients with clinically advanced peripheral vascular disease. Angiology 2008; 59: 198-202. 
40. van der Meer IM, Iglesias del Sol A, Hak AE, Bots ML, Hofman A, Witteman JC. Risk factors for progression of atherosclerosis measured at multiple sites in the arterial tree: the Rotterdam Study. Stroke 2003; 34: 2374-9.

41. Fowkes FG. Epidemiological research on peripheral vascular disease. J Clin Epidemiol 2001; 54: 863-8.

42. Gottsäter A. Managing risk factors for atherosclerosis in critical limb ischaemia. Eur J Vasc Endovasc Surg 2006; 32: 478-83.

43. Jaccard Y, Walther S, Anderson S, Tauber M, Kummer O, Baumgartner $\mathbf{R}$, et al. Influence of secondary infection on amputation in chronic critical limb ischemia. Eur J Vasc Endovasc Surg 2007; 33: 605-9.

44. Jude EB, Oyibo OA, Chalmers N, Boulton AJ. Peripheral arterial disease in diabetic and nondiabetic patients: a comparison of severity and outcome. Diabetes Care 2001; 24: 1433-7.

45. Abou-Zamzam AM Jr, Gomez NR, Molkara A, Banta JE, Teruya H, Killeen JD, et al. A prospective analysis of critical limb ischemia: factors leading to major primary amputation versus revascularization. Ann Vasc Surg 2007; 21: 458-63.
46. Virkkunnen J, Heikkinen M, Lepäntalo M, Metsänoja R, Salenius JP; Finnvasc Study Group. Diabetes as an independent risk factor for early postoperative complications in critical limb ischemia. J Vasc Surg 2004; 40: 761-7.

47. Taylor SM, Cull DL, Kalbaugh CA, Cass AL, Harmon SA, Langan EM 3rd, et al. Critical analysis of clinical success after surgical bypass for lower-extremity ischemic tissue loss using a standardized definition combining multiple parameters: a new paradigm of outcomes assessment. J Am Coll Surg 2007; 204: 831-9.

48. Taylor SM, Kalbaugh CA, Blackhurst DW, Hamontree SE, Cull DL, Messich HS, et al. Preoperative clinical factors predict postoperative functional outcomes after major lower limb amputation: an analysis of 553 consecutive patients. $J$ Vasc Surg 2005; 42: 227-35.

49. Taylor SM, York JW, Cull DL, Kalbaugh CA, Cass AL, Langan EM 3rd. Clinical success using patient-oriented outcome measures after lower extremity bypass and endovascular intervention for ischemic tissue loss. J Vasc Surg 2009; 50: $534-41$

50.Hill AB. The environment and disease: association or causation? Proc R Soc Med 1965; 58: 295-300. 